\title{
Tagungsbericht
}

Andreas Kraft*

\section{Tagungsbericht zum Transdisziplinären Kolloquium 2016: „Information und Wissen“}

Was ist Wissen - wie entsteht es, wie ändert es sich, wie wird es vermittelt? Fragestellungen, die nicht unbedingt neu, aber aus inter- und transdisziplinärer Perspektive aktueller sind denn je und von Wissenschaftlerinnen und Wissenschaftlern unterschiedlicher Disziplinen vom 21.4.-22.4.2016 am Fachgebiet Deutsch als Fremd- und Fachsprache der Technischen Universität Berlin eingehend diskutiert wurden. Zur Veranstaltung luden Thorsten Roelcke (Technische Universität Berlin), Matthias Ballod (Martin-Luther-Universität Halle-Wittenberg) und Tilo Weber (Technische Universität Liberec/MartinLuther-Universität Halle-Wittenberg) ein, unterstützt von Kristina Pelikan (Schweizerisches Tropen- und Public Health - Institut Basel/Technische Universität Berlin). Im durchgängigen Format der Tagung wurden einzelne Impulsvorträge der Referentinnen und Referenten im Rahmen von KnowledgeCafés aufgegriffen, um sowohl im Vorfeld aufgestellte Thesen als auch Fragestellungen intensiv diskutieren zu können.

Im Fokus der Veranstaltung stand der inter- und transdisziplinäre Austausch spezifischer Vorstellungen und Auffassungen von Information und Wissen. Ziel war es, exzellente Wissenschaftlerinnen und Wissenschaftler aus oft voneinander isolierten Fachdiskursen zusammenzubringen, um die Thematik aus unterschiedlichen wissenschaftlichen Perspektiven beleuchten und hinterfragen zu können. Von zentraler Bedeutung waren Fragestellungen wie „Was ist eigentlich Wissen?“, „Welche Wissenskonzeptionen präg(t)en die eigene Fachdisziplin?“, „Gibt es interdisziplinäre Schnittstellen von Wissenskonzeptionen?“ und „Wie lassen sich aus Gemeinsamkeiten und Unterschieden von Wissensauffassung(en) transdisziplinäre Fragestellungen und Forschungszweige formulieren?“.

Begrüßung und Auftakt erfolgte durch Thorsten Roelcke, Fachgebietsleiter der tagungsausrichtenden Universität, der den insgesamt 19 Teilnehmenden diverse Thesen bzw. Fragen zur Wissensgenerierung vorlegte. So unterschied er in seinem einleitenden Keynote-Vortrag „wissen“ von „informieren“, sowohl

*Corresponding author: Andreas Kraft, Technische Universität Berlin 
aus geschichtlicher als auch gegenwärtiger Perspektive, und ging hypothetisch davon aus, dass Sprachkompetenz eine Kompetenz der Wissensgenerierung sei und sich daraus unterschiedliche Gesichtspunkte und Fragestellungen ergeben, wie bspw.: „Die Rolle der Sprachkompetenz im Rahmen einer kognitiven Gesamtkompetenz“, „Das Wirken lingualer oder semiotischer Strukturen auf das Wissen“, „Einfluss der Sprachspezialisierung auf sprachliche Strukturen und Kompetenzen“, „Kognitive Verfahren der Wissensgenerierung“ oder „Das Verhältnis von Sprache und Erfahrung“, um nur einige zu nennen.

Der anschließende Vortrag von Gerd Antos (Martin-Luther-Universität Halle-Wittenberg) „Digitale Philologie und Hermeneutik: These gegen kommunikative Selbstevidenz“ griff u.a. die Fragestellung auf, welche Rollen heutzutage noch Geisteswissenschaftler spielen, wenn bereits alles digitalisiert und im Web abrufbar ist? So sei das Web semantisch zum Welttheater sprachlich erzeugter (Wissens-)Wirklichkeiten avanciert und habe sich zur selbstverstärkenden „Echokammer“ für verbreiteten Sinn und Unsinn entwickelt. Digitale Überkommunikation, Erlebnis- und Selbstkommunikation oder die digitale „Fake-Kultur“ sind nur einige von Antos genannten Entwicklungen des Strukturwandels der sprachlichen Kommunikation durch das Internet.

In seinen „Thesen zur internationalen Gesundheitsforschung“ ging Jakob Zinsstag (Schweizerisches Tropen- und Public Health - Institut Basel, Schweiz) im dritten Vortrag des Tages u.a. davon aus, dass die Internationale Gesundheit (IG) einen holistischen Ansatz verfolge, in dem akademisches und nicht-akademisches Wissen aufeinander bezogen werden und sich Forschung und soziale Interaktion gegenseitig positiv ergänzen würden. Die dafür notwendigen Prozesse bezeichnete Zinsstag als transdisziplinäre Forschung. Beispielhaft führte er ein Projekt zur interdisziplinären und humanmedizinischen Versorgung an und veranschaulichte daran den „One-Health“-Ansatz, der das Wechselverhältnis von Mensch, Tier und Umwelt beschreibt.

Konrad Ehlich stellte mit seinem Beitrag 13 Thesen rund um das Wissen auf, die u.a. europäische Wissenschaftskonzeptionen, Theorienentwicklungen und deren Auswirkungen auf semantische Potenziale, sprachliche Determinierung des Wissens-in-Bezug-auf-das-Wissen oder Strukturtypen des Wissens thematisierten.

Im Vortrag „Macht Information Sinn? Fünf vorbereitende Thesen zur Iteration des Unterschieds“ betrachtete Walther Ch. Zimmerli (HumboldtUniversität zu Berlin) „Information“ zunächst aus semantischer und pragmatischer Sicht und leitete daraus die verschiedenen Auffassungen von „Unterschied“ ab. So sei in pragmatischer Weise „Information“ Unterschied mit Bedeutung, wobei sich die sprachpragmatische Rekonstruktion von „Bedeutung“ 
wiederum durch Iteration der informationstheoretischen Bedeutung von „Unterschied“ vornehmen ließe. Nach Zimmerli sei Information Unterschied, der einen Unterschied mache: Wissen könne als kohärente Menge von Unterschieden des beobachtenden Subjekts aufgefasst werden und beschreibe auf dieser Ebene „Unterschied“ als „Sinn“. Daraus schlussfolgerte er, dass Information nur im Unterschied von Wissen und Nichtwissen Sinn mache, und resümierte abschließend, dass unsere Informationsgesellschaft keine Wissens-, sondern eine Nichtwissensgesellschaft sei.

Den letzen Beitrag des ersten Veranstaltungstages, „Zwei Perspektiven auf Wissen in den Transferwissenschaften“, präsentierten gemeinsam Kristina Pelikan (Schweizerisches Tropen- und Public Health - Institut Basel/ Technische Universität Berlin) und Tilo Weber (Technische Universität Liberec/Martin-Luther-Universität Halle-Wittenberg). Neben Ausführungen zum Wissen in der Fachkommunikation gingen sie speziell auf Wissensmanagement und Wissenstransfer ein und stellten fünf Thesen zum individuellen und kollektiven Wissen auf. So bewirke Wissenskommunikation zwar die Transformation von Wissen, jedoch nicht dessen Transfer. Offen bleibt dabei im Weiteren, wie Kollektive das Wissen ihrer Mitglieder prägen und sich das Verhältnis zwischen individuellem und kollektivem Wissen gestaltet.

Am zweiten Tag des Transdisziplinären Kolloquiums fasste Tilo Weber einleitend die Ergebnisse vom Vortag zusammen, woraufhin Uli Vilsmaier (Leuphana Universität Lüneburg) mit dem Thema „Wissen aus der Perspektive transdisziplinärer Nachhaltigkeitsforschung“ begann. Wissen sei für sie nicht nur durch Bewegtheit charakterisiert, sondern finde hier auch den Ort seiner Bestimmung. So versuche die transdisziplinäre Nachhaltigkeitsforschung Wissen hervorzubringen, das sich aus unterschiedlichen Wissens- und Erkenntniskulturen speise. Exemplarisch führte sie hierzu ein transdisziplinäres Forschungsprojekt an, in dem es um die nachhaltige Stärkung und Entwicklung mittels „Case-based Mutual Learning“ der kleinbäuerlichen Landwirtschaft Kolumbiens geht.

Günter Abel (Technische Universität Berlin), der im zweiten Beitrag des Tages „Das Wechselspiel von Wissen und Information“ anhand von 10 aufgestellten Thesen diskutierte, ging zunächst auf die Vielfalt unterschiedlicher Typen von Wissen und Information ein. Zwar korrelierten Wissen und Information, allerdings lägen hier Grenzen vor, da Wissen gegenüber Information einen epistemischen und normativen Mehrwert habe und mehr als eine bloße Aufsummierung von Daten und Informationen sei. Auch das Bewusstsein sei diesen Grenzen ausgesetzt, und es könne nicht davon ausgegangen werden, dass sich das Bewusstsein als ein Sammeln und Verknüpfen von verstreuten Informationen erkläre. 
Der anschließende Vortrag „Theoretische Grundlagen der Interdisziplinarität“ von Roland Posner (Technische Universität Berlin) führte einleitend Kriterien zur beruflicher und wissenschaftlicher Disziplinarität auf. Um wissenschaftliche Disziplinarität genauer definieren zu können, ging er auf ontologische, idealistische, logische, sozialwissenschaftliche wie genetische Ansätze ein und schlussfolgerte, dass die Kombination und Abgrenzung der Kriterien „Gegenstandsbereich“, „Perspektive“, „Methoden“, „Theorie“ und „Darstellungsmittel“ notwendige Bedingung dafür sei, um ein Tätigkeitsfeld als Disziplin im wissenschaftstheoretischen Sinne definieren zu können. Anhand der Linguistik zeigte er beispielhaft auf, wie diese Kriterien zueinander in Beziehung stehen und als theoretische Grundlage von Multi-, Inter- und Transdisziplinarität verstanden werden könnten.

Den letzten Beitrag der Tagung zum Thema „Wissen in der Theologie“ präsentierte Christian Schwarke (Technische Universität Dresden), der zunächst den Begriff des Glaubens und den eigentlichen Gegenstand der Theologie thematisierte. In seiner Darstellung vertrat Schwarke die These, dass naturwissenschaftliches und theologisches Wissen zwei Pole eines Kontinuums darstellten. Den prägenden Unterschied im Wissenserwerbsprozess spiele hierbei die konzeptionelle Rolle des Subjekts, was Schwarke zu der Frage führte, ob Wissen oder Strukturen des Wissenserwerbs generell transferierbar sind.

Zusammenfassend kann festgehalten werden, dass Information und Wissen aus ganz unterschiedlichen Wissenschaftsgebieten eingehend beleuchtet wurden und sich sowohl für die Teilnehmenden als auch für die Referentinnen und Referenten neue und bis dato unbekannte Herangehensweisen an die Thematik eröffneten. Trotz - oder aufgrund - der gewollt überschaubaren Teilnehmerinnen- und Teilnehmerzahl kam es im Rahmen der KnowledgeCafés zu einem intensiven inter- und transdisziplinären Wissensaustausch bei anregenden Diskussionsrunden, deren Ergebnisse separat publiziert werden sollen. Die fast familiäre Atmosphäre sowie der reibungslose Ablauf spiegelte die sehr gute Organisation der Veranstaltung wider.

Inhaltlich anknüpfend wird das nächste Transdisziplinäre Kolloquium im März/April 2017 in Halle stattfinden, um Antworten auf die abschließende Frage Schwarkes nach Transferierbarkeit des Wissens $\mathrm{zu}$ finden. Die Teilnahme ist unbedingt zu empfehlen.

Andreas Kraft

Veranstalter:

Prof. Dr. Thorsten Roelcke, Prof. Dr. Matthias Ballod, apl. Prof. Dr. Tilo Weber 


\section{Organisation:}

Kristina Pelikan M.A.

Referentinnen und Referenten (alphabetische Sortierung):

Prof. Dr. Günter Abel, Prof. em. Dr. Gerd Antos, Prof. Dr. Matthias Ballod, Prof. Dr. Dr. h.c. Konrad Ehlich, Kristina Pelikan M.A., Prof. em. Dr. Roland Posner, Prof. Dr. Thorsten Roelcke, Prof. Dr. Christian Schwarke, Prof. Dr. Ulli Vilsmaier, apl. Prof. Dr. Tilo Weber, Prof. Dr. Walter Zimmerli, Prof. Dr. Jakob Zinsstag 